\title{
APLICAÇÃO DE SISTEMA DE MEDIÇÃO DE RUGOSIDADE ON-LINE E SEUS BENEFÍCIOS*
}

Anderson Rosa Pereira ${ }^{1}$

\section{Resumo}

A constante busca pela excelência na qualidade do material produzido tem gerado demanda para métodos precisos e de determinação on-line das características do material. Os métodos hoje utilizados para coleta de dados de rugosidade superficial geram amostras randômicas ou concentradas no início ou fim da bobina, o que não necessariamente mostra com precisão a rugosidade de todo o material produzido, e impossibilitam uma intervenção mais direta, precisa e automática nos parâmetros de laminação, objetivando um melhor aproveitamento dos cilindros, redução de custos e controle sobre este importante parâmetro de qualidade. Esta publicação descreve o funcionamento e a aplicação do sistema de medição de rugosidade on-line EMGSORM 3plus no controle automático de rugosidade desenvolvido para um skin pass mill. Assim, a linha principal deste artigo mostra através de um exemplo prático a aplicação de um sistema de medição de rugosidade on-line e seus benefícios.

Palavras-chave: Medição de rugosidade on-line; Inspeção superficial; Controle automático de skin pass mill; Equipamento para garantia da qualidade.

\section{APPLICATION OF ONLINE ROUGHNESS MEASUREMENT SYSTEM AND ITS BENEFITS}

\begin{abstract}
The constant search for excellence in the quality of the material produced has generated a demand for precise methods as well as methods for on-line determination of the material features. The methods currently used for data collection of superficial roughness generates random or concentrated samples in the beginning or in the end of the coil, which it does not necessarily show with accuracy the roughness of the entire material produced, and make impossible a direct, accurate and automatic intervention in the rolling mill parameters, aiming a better use of the cylinders, cost reduction and control over this important quality parameter. This publication describes the functioning and application of the on-line roughness measurement system EMG-SORM 3plus in the automatic control of roughness developed for a skin pass mill. Therefore, the main point of this article shows through a practical example the application of an online roughness measurement system and its advantages.
\end{abstract}

Keywords: On-line roughness measurement; Surface inspection; Skin pass mill automatic control; Quality assurance equipment.

1 Engenheiro eletricista, coordenador de vendas, departamento de engenharia, EMH, Belo Horizonte, MG, Brasil. 


\section{INTRODUÇÃO}

O desempenho das modernas linhas de processo da indústria siderúrgica, por exemplo, plantas de galvanização por imersão a quente (CGLs), assim como os altos requisitos de qualidade impostos pelos usuários do aço laminado, requerem, cada vez mais, um nível de acompanhamento on-line do processo. O monitoramento completo das principais características do material em todo o processo produtivo é necessário para alcançar e manter um alto nível de qualidade. Enquanto os sistemas de engenharia têm estabelecido um alto grau de controle automático do processo, 0 skin pass mill é ainda controlado manualmente pelo operador. Isto é devido ao fato de que até agora os parâmetros do processo que podem ser influenciados durante a laminação, por exemplo a rugosidade, são determinados utilizando o rugosímetro, com medições aleatórias ou feitas no topo/base da tira, ou com um atraso considerável quando as medições são feitas no laboratório.

O sistema on-line EMG-SORM 3plus fornece ao operador da linha (galvanização por imersão a quente, recozimento contínuo ou skin pass mill) informações confiáveis e contínuas sobre a rugosidade ao longo do comprimento e da largura da tira. $\mathrm{O}$ significado estatístico, bem como a aplicabilidade dos resultados medidos é claramente superior quando comparado com a medição tradicional por rugosímetro.

Somente a utilização de técnicas de medição on-line possibilita a representação transparente da rugosidade realmente produzida, permitindo um controle de malha fechada do processo de laminação no skin pass mill. Quando este método de controle é usado, a variação típica de rugosidade ao longo do comprimento da tira é fortemente reduzida.

O relatório a seguir descreve o princípio de funcionamento do sistema medidor de rugosidade on-line EMG-SORM 3plus, a experiência operacional de um cliente que usou o equipamento para controlar o SPM, bem como alguns outros benefícios da medição de rugosidade on-line.

\section{PRINCÍPIO DE FUNCIONAMENTO DO EMG-SORM 3plus}

O princípio de funcionamento do SORM é a medição angular do feixe de luz difusa refletida (figura 1). Um feixe de laser é direcionado para a superfície do material. $\mathrm{O}$ diâmetro máximo do feixe de laser é $6 \mu \mathrm{m}$. Basicamente a superfície do material é feita de uma série de pequenas faces, isto é, pequenas saliências ou reentrâncias que a caracterizam.

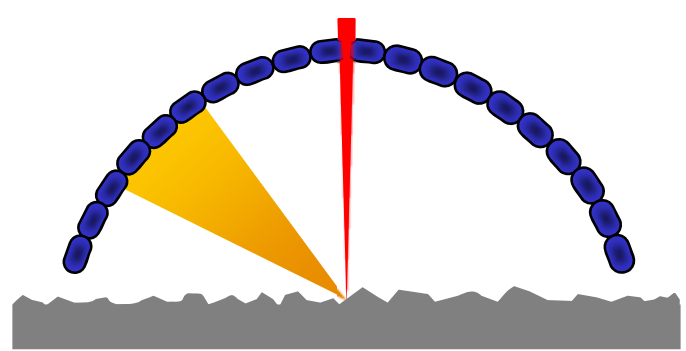

Figura 1. Princípio de medição do SORM 3plus (arranjo ótico)

Cada face reflete pelo menos uma pequena porção de luz, como um espelho. Um sensor com abertura total de $134^{\circ}$ capta o feixe de luz difusa refletida e gera um sinal proporcional a média do ângulo de reflexão (figura 1). 
A medição da face é feita a cada $5 \mu \mathrm{m}$ sobre um determinado número de pontos de medição que são dispostos consecutivamente (figura 2), e o comprimento máximo de medição é de $300 \mathrm{~mm}$. A mudança de superfície do material gera uma gama de valores que representa a rugosidade superficial através da disposição dos ângulos.

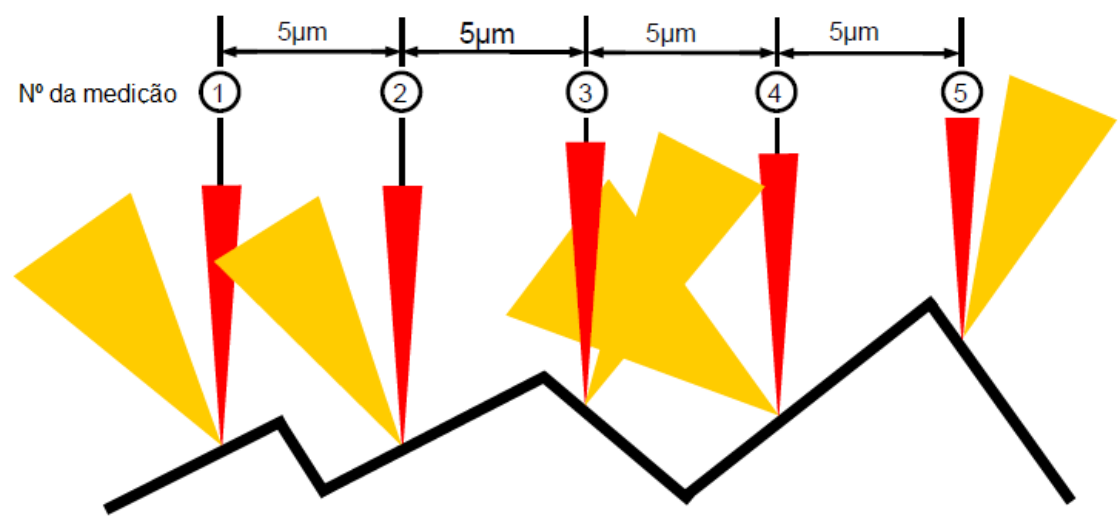

Figura 2. Princípio de funcionamento da obtenção de dados

O igual espaçamento entre os pontos de medição bem como os ângulos medidos permitem o cálculo do perfil da rugosidade na superfície do material.

A fim de calcular o perfil de rugosidade da superfície, as várias medidas de altura são integradas ao longo do comprimento medido. Com base no perfil obtido, os parâmetros de rugosidade (Por exemplo: Ra, RPc, Rz) são determinados de acordo com as normas aplicáveis (filtros, algoritmos). A figura 3 é uma representação esquemática do cálculo do parâmetro de rugosidade.

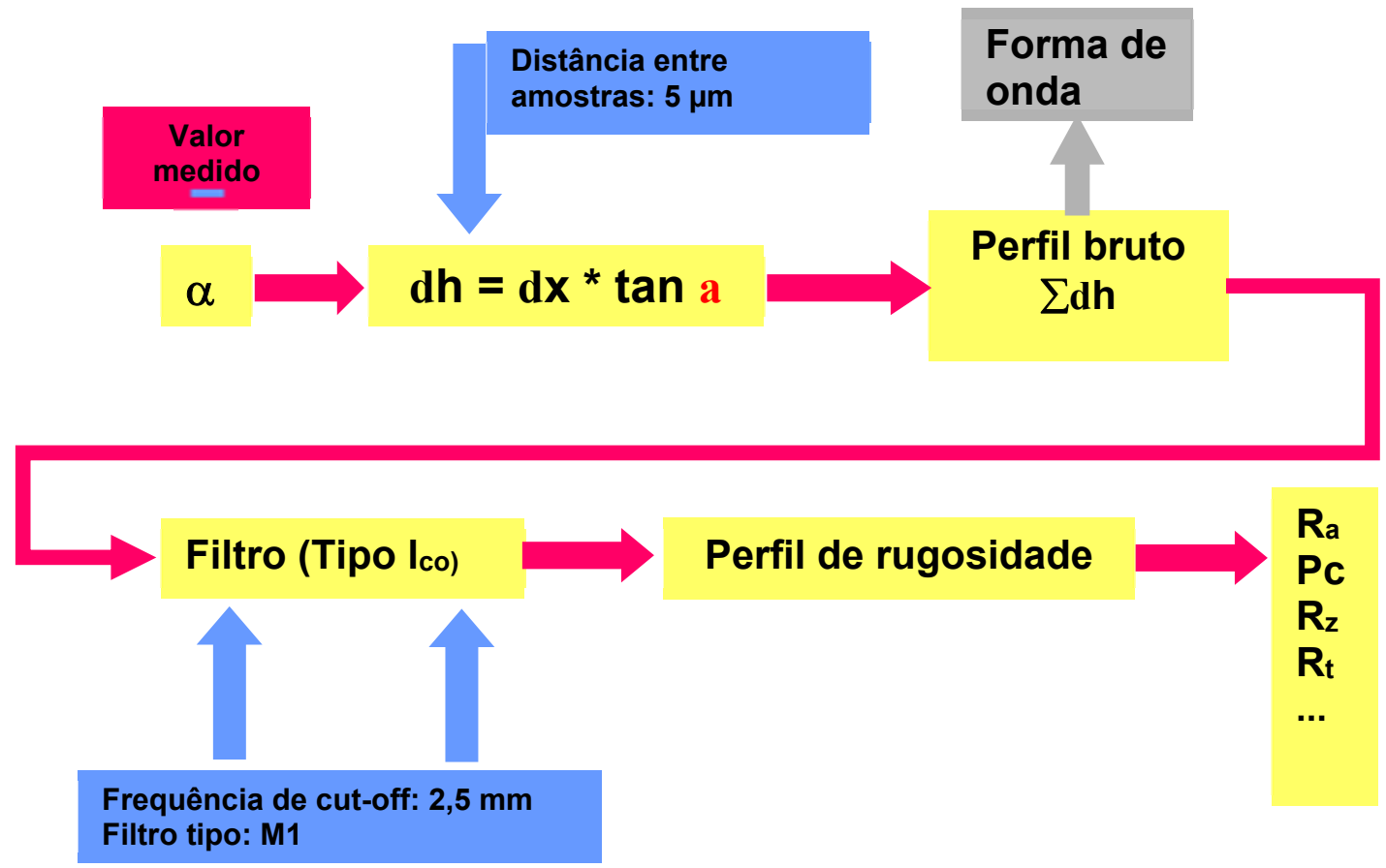

Figura 3. Diagrama de bloco dos parâmetros de cálculo de rugosidade 


\section{CONTROLE MANUAL DA CADEIRA DE LAMINAÇÃO DO SPM}

O laminador skin pass é um re-laminador de material recozido com um pequeno grau de deformação (grau de laminação do skin pass), tipicamente dentro de uma faixa de 0.5 a $2 \%$. Basicamente o objetivo do skin pass é atingir o correto limite de escoamento e as características superficiais desejadas.

A passagem pelo SPM muda as características do material visando modificá-lo para definir o limite de escoamento que até então estava instável. Isto serve para melhorar o comportamento de fluxo durante a estampagem profunda e prevenir as indesejáveis linhas de estresse.

A planicidade da tira é uma importante propriedade para as empresas que realizam processamento posterior do material. Isto porque bons valores de planicidade permitem uma operação sem problemas ou interrupções nas linhas de produção.

Por fim, a aplicação da rugosidade desejada na superfície da tira vai, por exemplo, melhorar o rendimento do processo de estampagem profunda, bem como melhorar a adesão das tintas.

Atualmente a cadeira do laminador do skin pass, em uma linha de galvanização por imersão a quente, é controlada manualmente por um operador que recebe os valores definidos para ajuste da cadeira a partir de um banco de dados. Este valor padrão é manualmente ajustado pelo operador após a passagem do ponto de solda, no início da bobina. A figura 4 mostra o processo de controle manual.

Os valores de ajuste da cadeira de laminação são salvos em um banco de dados (força de laminação, tensão da tira, alongamento)

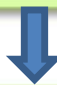

Usando esses ajustes o operador inicia a laminação no skin pass depois do ponto de solda ter passado, de acordo com os dados

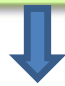

Para uma tensão de tira e grau de alongamento skin pass definidos, a força de laminação é ajustada até o alongamento ser atingido

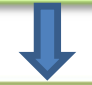

A rugosidade é medida usando o rugosímetro no final da bobina ou na bobina atual

O operador corrige a força de laminação, alongamento no skin pass e tensão da tira para adequar aos requisitos do pedido

Figura 4. Controle manual da cadeira do skin pass mil pelo operador

O princípio de controle manual da cadeira do skin pass mil gera desvantagens devido ao atraso de tempo entre a laminação real e a captura dos parâmetros alvo (ex: Rugosidade, limite de escoamento). Estas desvantagens são:

- Materiais com propriedades instáveis ou indefinidas (rugosidade e limite de escoamento) no início da bobina, quando o laminador skin pass está reajustando a força de laminação até que o grau do skin 
pass seja alcançado. Este efeito é especialmente sentido quando existe mudança de material.

- O operador não tem nenhuma informação fundamentada depois das mudanças de parâmetros de laminação do skin pass.

- Muitas vezes a medição de rugosidade é feita em uma placa de teste no topo ou na base da tira diretamente na linha. Para medições no topo da tira os resultados somente serão confiáveis se as bobinas produzidas tiverem as mesmas características, caso contrário as medições serão feitas na faixa de ajuste do skin pass. Assim, medições feitas nessas áreas são geralmente problemáticas e podem levar a erros.

- Medições feitas na seção de saída usando rugosímetro não fornecem confiabilidade estatística, principalmente devido ao tamanho do acumulador não há tempo suficiente para fazer variadas medições. Além disso, a variação na tensão da tira pode levar a resultados incorretos.

Na prática, no laminador skin pass, a obtenção da rugosidade necessária muitas vezes contradiz, do ponto de vista do material, o alongamento desejável. A supervisão contínua das propriedades do material possibilita uma considerável margem de manobra para trabalhar com a rugosidade especificada e economizar a utilização dos rolos de trabalho.

A fim de analisar o impacto das mudanças de parâmetros em relação ao laminador skin pass, uma bobina de teste foi usada em uma linha de galvanização por imersão a quente de um cliente que não terá o nome divulgado neste trabalho (Aço IF, grade DX54D+Z; dimensões: $0,8 \mathrm{~mm} \times 1520 \mathrm{~mm}$ ). Nesta bobina, o alongamento no skin pass variou em relação a tensão do material ao longo do comprimento da tira. A figura 5 mostra a rugosidade média $\mathrm{Ra}$ em relação ao alongamento no skin pass para duas diferentes tensões de tira.

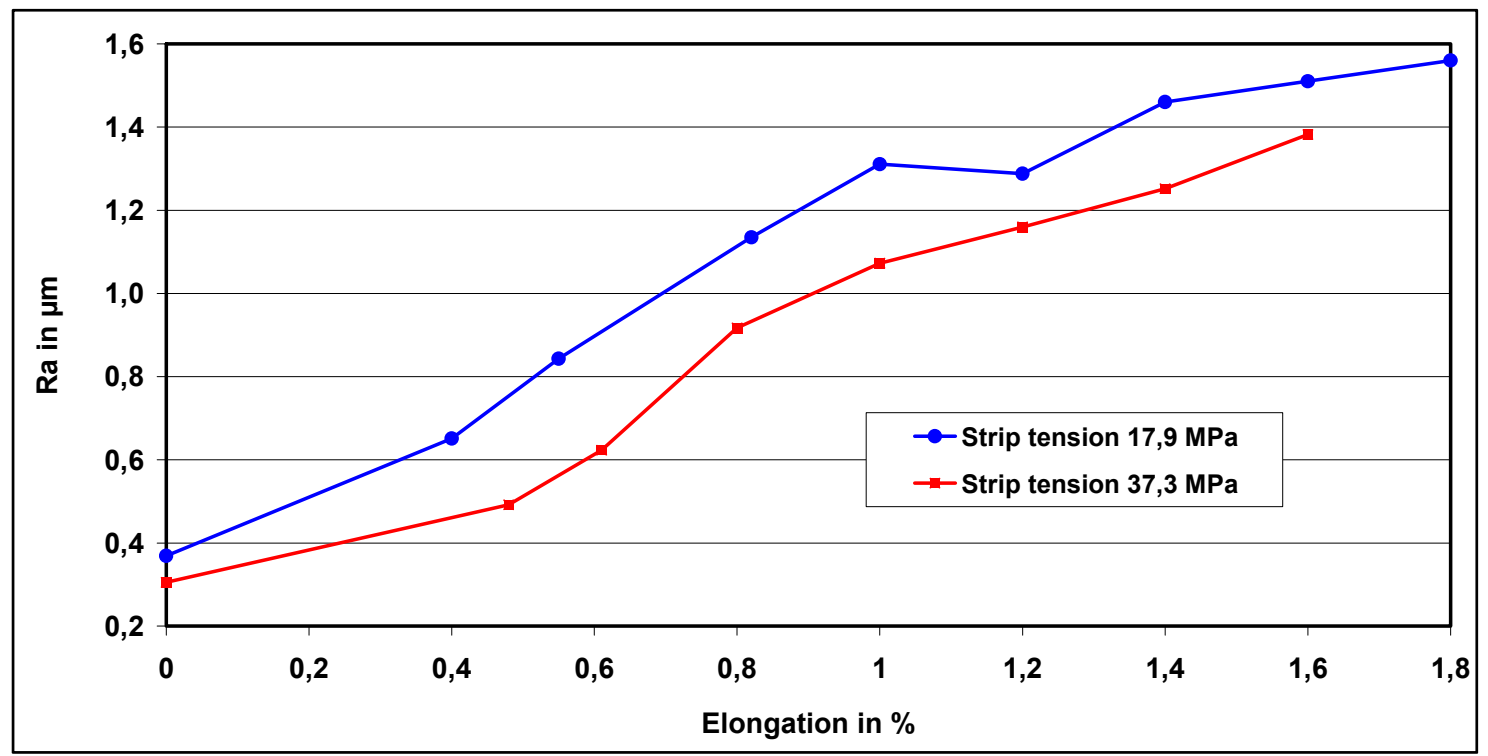

Figura 5. Média aritmética de rugosidade Ra em relação ao alongamento

Como era esperado o valor Ra é aumentado com uma tensão de tira constante de forma quase diretamente proporcional ao alongamento e por consequência a força de laminação. A medida que a tensão da tira é aumentada a força de laminação, e 
por consequência o valor de Ra é reduzido enquanto o alongamento permanece o mesmo. O aumento médio é:

- $0.14 \mu \mathrm{m}$ por $0.2 \%$ de alongamento para uma tensão de tira de $17.9 \mathrm{Mpa}$,

- $0.15 \mu \mathrm{m}$ por $0.2 \%$ de alongamento para uma tensão de tira de $37.3 \mathrm{Mpa}$,

- Valor médio: $0.135 \mu \mathrm{m}$ por $0.2 \%$ de alongamento.

Outro problema no laminador skin pass é a definição da correta força inicial de laminação depois de uma mudança de material. A figura 6 mostra um exemplo de um desvio típico da força de laminação especificada depois de uma mudança de material. Somente depois de $250 \mathrm{~m}$ a cadeira de laminação do skin pass atinge uma força de laminação de $5400 \mathrm{kN}$ onde o alongamento ajustado do skin pass, por exemplo $1.0 \%$, é atingido (força de laminação especificada: $2200 \mathrm{kN}$ ). Ao mesmo tempo, Ra na face superior da tira muda até $0.50 \mu \mathrm{m}$, exemplo de $\mathrm{Ra}=1.25 \mu \mathrm{m}$ no início da tira para $\mathrm{Ra}=1.72 \mu \mathrm{m}$ após $250 \mathrm{~m}$ processados. A rugosidade $\mathrm{Ra}$ na face inferior da tira também muda, por exemplo $0.50 \mu \mathrm{m}$. A tensão da tira varia somente ao longo dos primeiros $50 \mathrm{~m}$, e depois de um comprimento de aproximadamente $20 \mathrm{~m}$ já atingiu o valor parametrizado de aproximadamente $70 \mathrm{kN}$. Na tabela seguinte a cadeira do laminador skin pass e os parâmetros de rugosidade estão compilados.

Tabela 1. Rugosidade e parâmetros do skin pass em caso de especificação de força de laminação indefinida (figura 6)

\begin{tabular}{|l|l|l|l|}
\hline Dimensões da tira & $\begin{array}{l}\text { Comprimento } \\
\text { de tira: } 5 \mathrm{~m}\end{array}$ & $\begin{array}{l}\text { Comprimento } \\
\text { de tira: 256m }\end{array}$ & Diferença \\
\hline Alongamento em \% & 0.27 & 1.00 & 0.73 \\
\hline Força de laminação em kN & 2175 & 5827 & 3652 \\
\hline Ra na face superior em $\mu \mathrm{m}$ & 1.25 & 1.72 & 0.47 \\
\hline Ra na face inferior em $\mu \mathrm{m}$ & 1.43 & 1.95 & 0.52 \\
\hline
\end{tabular}




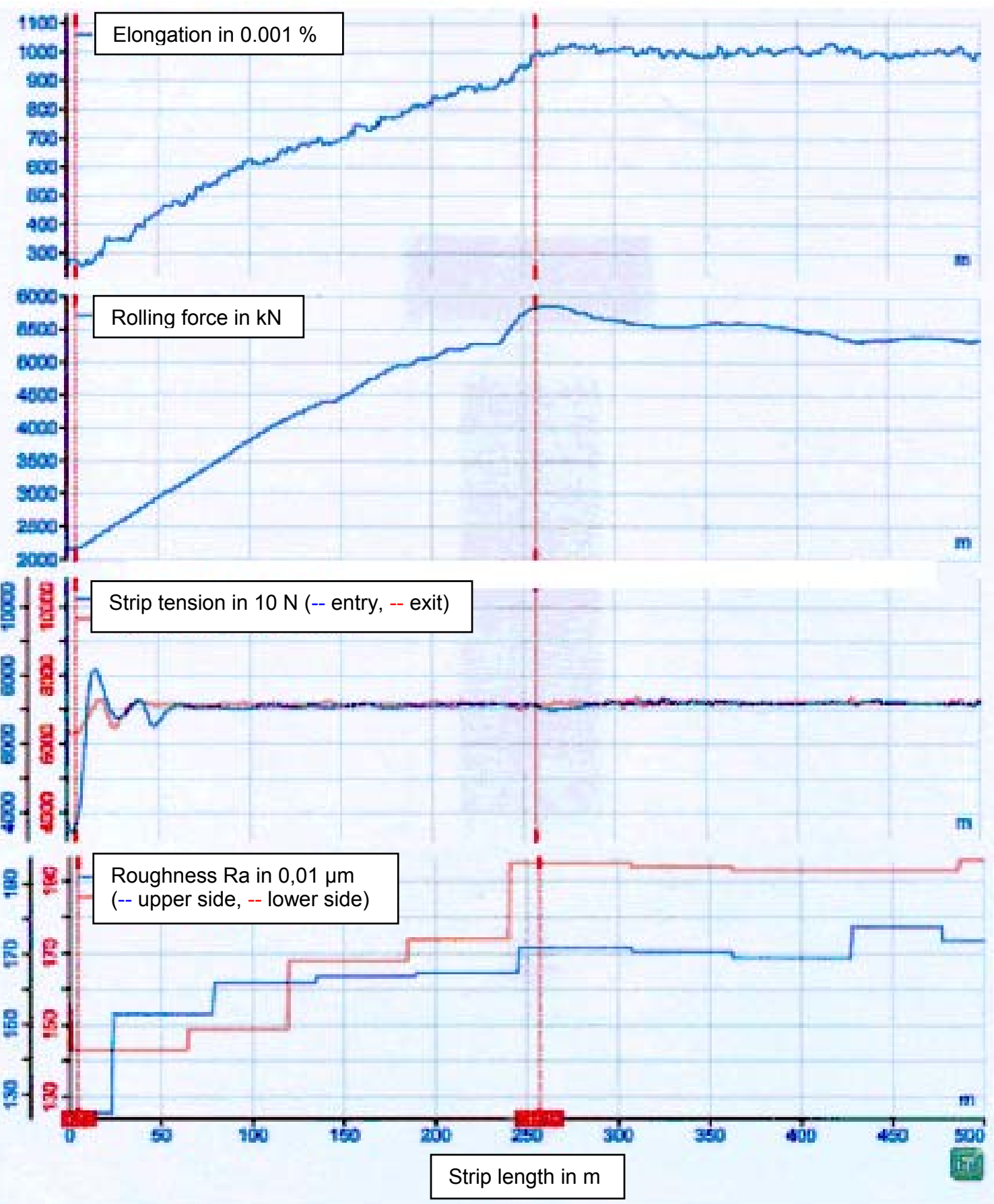

Figura 6. Comportamento de ajuste da cadeira do skin pass em caso de uma força de laminação especificada incorreta no início da bobina

\section{CONTROLE DO SKIN PASS COM A FERRAMENTA EMG-SORM 3PLUS}

O cliente não terá o nome divulgado por questões comerciais, contudo é usuário do SORM 3plus desde 2002, está localizado na Alemanha e, neste caso, o equipamento está instalado em uma linha de galvanização por imersão a quente CGL;

A instalação do equipamento medidor de rugosidade on-line no SPM da CGL teve os seguintes objetivos: 
- Após o ajuste do SPM ou uma vez que o ponto de solda passe através do sistema com mudança subsequente das especificações, a força de laminação e o valor da tensão da tira devem garantir a rugosidade bem como o alongamento, para atender às necessidades dos clientes.

- Uma vez que os valores on-line medidos pelo SORM 3plus (distância entre o skin pass e o SORM: $55 \mathrm{~m}$ ) estão disponíveis, o controle de rugosidade é ativado.

- Uma vez que o controle de rugosidade foi ativado, o laminador trabalha com base no controle de força de laminação fundamental usando a força total de laminação como um valor ajustado.

- A limitação na gama de ajuste é determinada pelo valor correspondente ao limite superior da força de laminação (para ser definida pelo operador, como antes).

A tarefa do sistema de controle é atingir a rugosidade necessária o mais rapidamente possível uma vez que a solda passe pelo medidor e manter a rugosidade controlada ao longo do comprimento da bobina. $\mathrm{O}$ algoritmo de controle básico é integrado pela definição de força de laminação e tensão da tira predefinida.

A ativação da força de laminação pré-ajustada e tensão da tira respectivamente, é exclusivamente fornecida pelos chamados "guias" de modo que a série de bobinas com controle de rugosidade ativada pode ser produzida sem intervenções manuais adicionais. Alterações dos valores parametrizados pelo operador tem o mesmo efeito da seleção de um novo valor parametrizado para tensão da tira ou potência de laminação. O cálculo dos valores pré-ajustados é baseado em modelos estatísticos que são adaptados sob memórias de longo e curto prazo por conta da força de laminação, rugosidade da tira, alongamento no skin pass, tensão da tira, velocidade da tira, material e tipo de rolo.

O operador seleciona o valor de rugosidade definido ou, como alternativa, este valor é fornecido a partir de um sistema de banco de dados de nível superior.

O valor real para o controle de rugosidade é a rugosidade atual média Ra medida pelo SORM onde os valores das bordas não são considerados em caso de operação transversal.

O valor definido para o controle é a soma da força de laminação definida e a força de laminação calculada pelo algoritmo de controle. O rolo de bending ou a compensação dos rolos de trabalho permanecem completamente inalterados. Se a força de laminação máxima for excedida ou o alongamento máximo ou mínimo de laminação do skin pass não for respeitado o controle é desativado. O ajuste de força de laminação é realizado com base em um controle PI levando em consideração o atraso de fase que é gerado devido a distância entre a cadeira do skin pass e a unidade de medição SORM. Este controle garante a produção de uma tira com rugosidade superficial constante Ra dentro de uma janela de tolerância que pode ser ajustada ao longo do comprimento da tira.

A figura 7 apresenta um exemplo de rugosidade, alongamento no skin pass e força de laminação como uma função da largura da tira em modo de controle de rugosidade. $\mathrm{O}$ valor parametrizado de $\mathrm{Ra}=1.4 \mu \mathrm{m}$ é atingido sem sobrecargas $\mathrm{e}$ mantido em todo o comprimento da tira com uma variação menor que $+/-0.2 \mu \mathrm{m}$. No modo de controle, as mudanças ocorrem para um valor parametrizado de força de laminação de até 200 kN sem sobrecargas. 


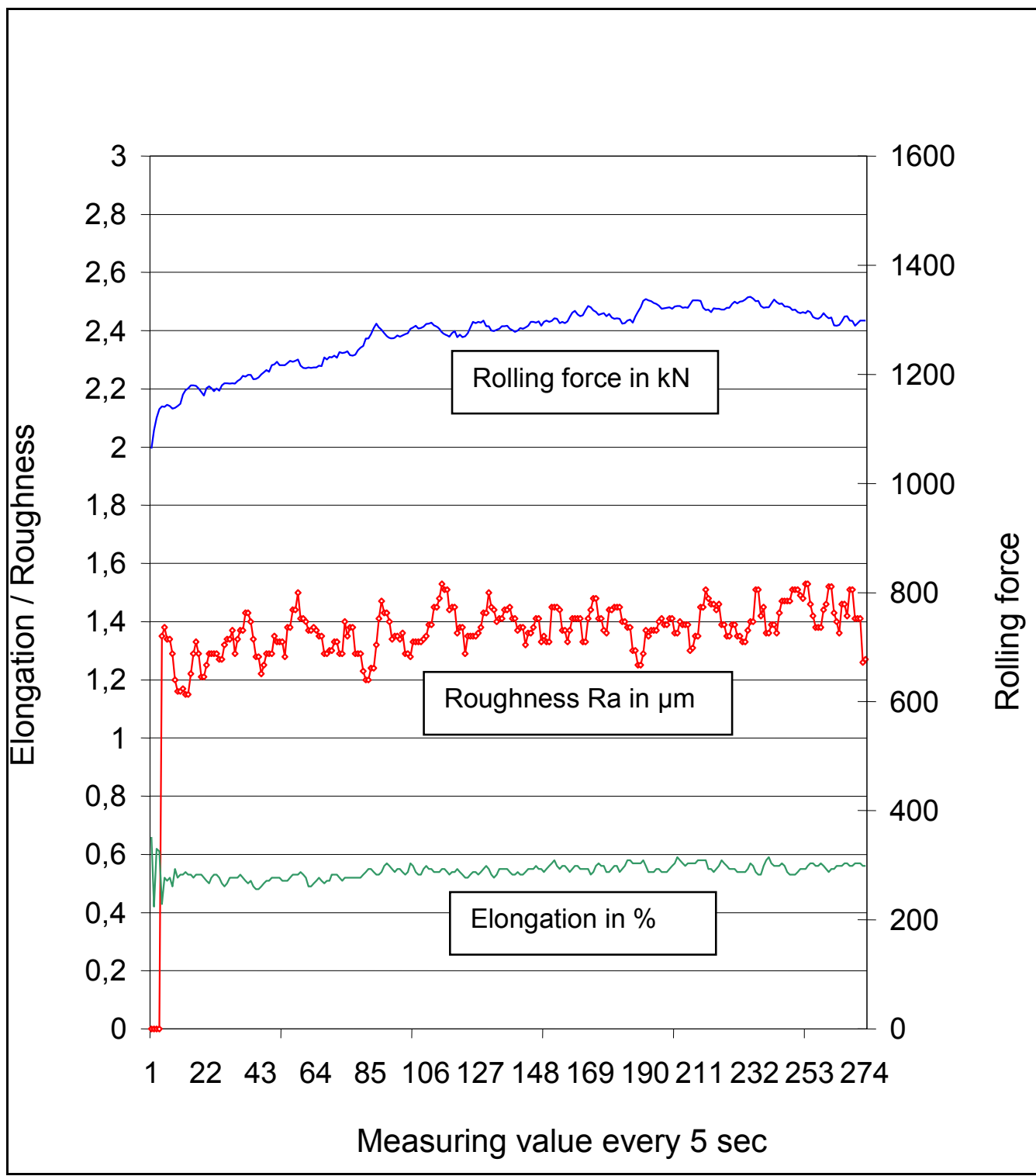

Figura 7. Parâmetros da cadeira do skin pass para controle de rugosidade ativa

Usando o controle de rugosidade, a variação de rugosidade observada ao longo do comprimento da tira foi claramente reduzida. Assim, a taxa de reclamação oriunda de defeitos ligados a rugosidade caíram drasticamente.

\section{CONCLUSÃO}

Portanto, o controle da cadeira do laminador skin pass no processo de acabamento de laminados a frio é hoje em dia realizado somente de forma não automatizada, por exemplo, o operador na planta ajusta os valores necessários de forma manual. A operação automática não é possível por causa do falta de tecnologia para medições apropriadas. O sistema medidor de rugosidade on-line SORM 3plus produzido pela EMG Automation, torna o controle em malha fechada de um Skin pass mill possível. O cliente usuário da tecnologia apresentada, fonte dos dados divulgados neste trabalho, implementou seu próprio conceito de controle de skin pass mill em uma linha de galvanização por imersão a quente com base nos dados gerados pelo SORM 3plus. Com o novo método de "controle de rugosidade" usado para operação da cadeira do skin pass, as variações de rugosidade ao longo do comprimento da 
tira puderam ser substancialmente reduzidos. A taxa de reclamação caiu e um maior controle sobre o parâmetro rugosidade pôde ser implantado.

Isto mostra que os métodos de medição on-line geram maior conhecimento sobre as linhas de produção, bem como sobre o material produzido, possibilitando um melhor controle de qualidade e redução nos custos de produção.

\section{BIBLIOGRAFIA}

1 Irle Matthias, Dornseifer Thomas. Control of the skin pass mill stand in a hot-dip galvanizing plant using on-line roughness measurement and on-line measurement of material properties, 2005:1-15.

2 SKIN-PASS MILLS for a perfect finish. 2012. [acesso em 01 abr. 2015];01:04-09. Disponível em: https://www.sms-siemag.com/download/W6_7_304E_SkinPass_Mills_References.pdf 